Methods: GLC mice were developed on a mixed genetic background (1290la/ Hsd-C57BL/6) by Cre-technology using LysM-promotor to knockout the Pggt1b gene in Mø[2]. CD4 ${ }^{+}$cells were isolated from spleen and lymph node (LN) of 16 weeks-old mice ( $G L C n=7$, wt $n=5$ ) expected to have high prevalence of arthritis. RNA was extracted to measure expression of the Rho proteins and signature genes to characterize differences in Th-subtypes and migration abilities of CD4 ${ }^{+}$ cells between GLC and wt mice. Furthermore, Illumina RNAseq analyzed the transcriptome of LN CD4 ${ }^{+}$cells. In a separate experiment we treated GLC mice with CTLA4-FP $(n=12)$ or PBS $(n=11)$ for 20 weeks from the age of 5 weeks. Rationale was to disrupt Mø/T cell contact to prevent arthritis. To study Rho-protein dependent phenotype in human RA, we performed RNAseq of sorted CD4 ${ }^{+}$ cells of RA patients.

Results: RNAseq showed that $\mathrm{CD}^{+}$cells in LN of GLC mice had IFN- $\gamma$ dependent cytotoxic profile and upregulated numerous pro-inflammatory genes including Eomes, Cxcr3, Tigit, Tnfsf10, II-1rl1, Stat1, Jak3, Irf7, Irf5, Ptpn13. Furthermore, the over-represented genes often depended on the IRF family in their transcription.

GLC mice overexpressed Cdc42 and Rac1 in spleen CD4 ${ }^{+}$compared to wt $\left(p=0.005\right.$ and $p=0.048$ resp.). Spleen GLC CD4 ${ }^{+}$cells had higher levels of $\alpha 5 \beta 1$ and $\alpha 2 \beta 2$ integrins, strongly correlating to $\operatorname{Cdc} 42(r=0.61 p=0.0027$ and $r=0.50, p=0.018)$ and arthritis $(r=0.64, p=0.0015$ and $r=0.69, p=0.0004)$. Importantly, Cdc42, Rac1, and RhoA were higher expressed in $\mathrm{LN} \mathrm{CD4}{ }^{+}$compared to spleen $(p=0.016, p=0.031$ and $p=0.016)$. In addition, Itg $b 1$ coding for $\beta 1$ integrin, was upregulated in GLC CD4 ${ }^{+}$cells of both spleen and LN ( $p=0.003 p=0.03$, resp.), suggesting Rho proteins are important for migration of $\mathrm{CD}^{+}$cells to the joint draining $\mathrm{LN}$ and for arthritis development. $\mathrm{CD} 4^{+}$cells that migrated to the LN had high proportion of Foxp $3^{+}$cells. This also correlated to the expression of Itgb1 ( $\mathrm{r}=0.84, \mathrm{p}=0.0012)$ presenting a plausible mechanism for increased influx of Tregs into joints. Several observations are in favor of this notion. First, GLC mice expressed more Foxp3 in LN compared to spleen $C D 4^{+}$cells $(p=0.016)$. Second, transcription of Foxp3 in LN CD4 ${ }^{+}$cells was higher in GLC mice compared to wt $(\mathrm{p}=0.015)$. Third, this high Foxp3 coexisted with low transcription of Lef1 $(p=0.03)$, required for Treg immunosuppression. Last, Foxp3 correlated negatively to both Lef1 $(r=-0.72, p=0.017)$, and its cofactor Tcf1 $(r=-0.75$, $\mathrm{p}=0.01$ ).

CTLA4-FP reduced inflammation in GLC mice evident as lower IFN- $\gamma$, IL-6 and TNF- $\alpha$ production ( $p=0.0002, p<0.0001$ and $p<0.0001$ resp.) and the number of CD2 $5^{+} \mathrm{CD} 4^{+}$cells in spleen $(p=0.027)$. In contrast, we observed increased IL-17A production $(p=0.056)$. However, CTLA4-FP treatment did not affect migration of $\mathrm{CD}^{+}$cells enriched with Rho-protein into draining LN nor alleviate arthritis.

Similar to the GLC mice, CD4 ${ }^{+}$cells of RA patients with high expression of RhoA, Rac1 and Cdc42 demonstrated enrichment for Th1 signature genes including IFNG, TBX21, Eomes, IL2RA, IL2RB, IL12RB2, TNF, IL18RAP (all, adj. p<0.05). Conclusion: This study shows that accumulation of Rho-proteins in $\mathrm{CD} 4^{+}$cells results in pro-inflammatory IFN- $\gamma$ dependent phenotype in mice and human RA. Accumulation of RhoA, Rac1 and Cdc42 proteins trigger the migration of CD4 ${ }^{+}$ cells into joint draining $L N$ and facilitates arthritis. Inhibiting $M \emptyset / T$ cell contact in GLC mice did not suffice to prevent migration of Rho-protein expressing cells and arthritis

REFERENCES:

[1] Khan, O.M., et al. J Clin Invest, 2011. 121(2): p. 628-39.

[2] Akula, M.K., et al. Nat Commun, 2019. 10(1): p. 3975.

Disclosure of Interests: None declared

DOI: 10.1136/annrheumdis-2021-eular.1323

\section{OP0023 $\quad$ GENERATION OF PT101, A HIGHLY SELECTIVE IL-2 MUTEIN FOR TREATMENT OF AUTOIMMUNE DISEASES}

J. Visweswaraiah ${ }^{1}$, E. Sampson ${ }^{2}$, P. Petaipimol ${ }^{1}$, A. Monsef ${ }^{3}$, K. Kis-Toth ${ }^{4}$, K. L. Otipoby ${ }^{2}$, J. Sundy ${ }^{3}$, N. Higginson-Scott ${ }^{1}$, J. Viney ${ }^{5} .{ }^{1}$ Pandion Therapeutics, Biotherapeutics, Watertown, United States of America; ${ }^{2}$ Pandion Therapeutics, Immunology, Watertown, United States of America; ${ }^{3}$ Pandion Therapeutics, Clinical, Watertown, United States of America; ${ }^{4}$ Pandion Therapeutics, Translational Sciences, Watertown, United States of America; ${ }^{5}$ Pandion Therapeutics, $R \& D$, Watertown, United States of America

Background: Regulatory T cells (Tregs) play a critical role in immune homeostasis and are dysfunctional in many autoimmune diseases. Interleukin 2 (IL-2) drives the proliferation and function of Tregs. via the heterotrimeric IL-2 receptor (CD25/CD122/CD132). As a result, CD25 loss-of-function in mice is associated with Treg deficiency and widespread autoimmunity. Low dose IL-2 is being evaluated for treatment of autoimmune diseases and has been shown to expand Tregs, however it has a narrow selectivity window before activating conventional T cells and natural killer cells. To enhance IL-2 selectivity and improve its therapeutic utility for activating and expanding Tregs, mutations can be introduced that reduce CD122/CD132 affinity thus creating a dependency on CD25 binding for signaling through CD122/CD132 upon IL-2 facilitated CD25/CD122/CD132 trimer formation.

Objectives: To generate a highly selective IL-2 mutein that activates and expands Tregs selectively that can be used for treatment of autoimmune diseases

Methods: Using a structure guided approach, we introduced mutations in IL-2 that significantly decreased CD122 binding affinity in addition to other mutations that increased CD25 binding affinity. Finally, we explored additional mutations, format, orientation, and linker lengths to generate the most potent, selective molecule with drug-like manufacturability. These structure activity relationship efforts culminated in the generation of PT101, a mutant IL-2 Fc fusion that is selective in activating and expanding Tregs in vitro and in vivo.

Results: PT101 selectively induced STAT5 phosphorylation in human and cynomolgus monkey Tregs in vitro. In humanized NOD-scid IL-2Rg-null (NSG) mice and cynomolgus monkeys, administration of PT101 dose-dependently and selectively expanded Tregs without significant effects on other immune cell types, and without eliciting proinflammatory cytokine production. The Tregs from PT101-dosed humanized mice have increased expression of FOXP3 and CD25, suggesting enhanced function and stability. In a Phase 1a single ascending dose clinical trial, PT101 was well-tolerated and selectively expanded total Tregs, with a mean maximal increase of up to 3.6-fold over baseline in healthy volunteers. There was no evidence of expansion of natural killer cells nor pro-inflammatory conventional T cells at any of the doses studied.

Conclusion: PT101 selectively activated and expanded Tregs without significant effects on other immune cell types, and without eliciting proinflammatory cytokine production. These Tregs have enhanced function and stability as seen by increase in expression of FOXP3 and CD25 in these cells. PT101 maintained selectivity in Phase 1 a clinical trial with no evidence of expansion of natura killer cells nor pro-inflammatory conventional $T$ at any dose studied. A Phase $1 \mathrm{~b} / 2 \mathrm{a}$ clinical trial in patients with ulcerative colitis and a Phase 2 clinical tria in patients with systemic lupus erythematosus are planned to further evaluate PT101.

Disclosure of Interests: None declared

DOI: 10.1136/annrheumdis-2021-eular.2097

\section{OP0024 \\ DECREASED LEVELS OF T FOLLICULAR HELPER (CD4+CXCR5+) CELLS AND CD27+CD38+ AND CD27+CD38- B CELLS IN ANKYLOSING SPONDYLITIS PATIENTS CORRELATE WITH MARKER OF INFLAMMATION}

H. Forsblad-D'elia ${ }^{1}$, U. Hellman ${ }^{2}$, A. Kumar ${ }^{2}$, K. Lejon ${ }^{3} .{ }^{1}$ Institute of Medicine, Department of Rheumatology and Inflammation Research, Gothenburg,

Sweden; ${ }^{2}$ Department of Public Health and Clinical Medicine, Umeå University, Umeå, Sweden; ${ }^{3}$ Department of Clinical Microbiology, Infection and Immunology, Umeå University, Umeå, Sweden

Background: The role of different lymphocyte subsets in ankylosing spondylitis (AS) is still to be elucidated. It has previously been reported contradictory data concerning the levels of T Follicular Helper (TFH) cells and differentiated B cells in peripheral blood of AS patients. In addition, the connection to disease related parameters is still to be fully revealed.

Objectives: The purpose of this study was to investigate the level of CD4+TFH cells and CD27+CD38+/CD38- B cells in patients with AS from northern Sweden and to compare the levels with age and sex-matched controls. We also studied associations between these cell subsets and disease related factors.

Methods: Peripheral blood mononuclear cells (PBMSc) from a cohort of 50 patients with AS from Region Västerbotten (mean age $52 \pm 9.1$ years, 33 (66 \%) men, 50 (100\%) HLAB27 positive) and 50 pair wise matched blood donor controls (mean age $54 \pm 8.8$ years, $33(66 \%)$ men) were stained with a combination of antibodies allowing for the detection of CD27, CD38, CD19, CD3, CD4 and CXCR5 markers and analyzed by flow cytometry. In addition, the patient with AS were examined with spinal $x$-ray for radiographic alterations assessed with mSASSS. CRP and ESR were measured and physical function and disease activity were registered with BASMI and BASFI respectively ASDAS-CRP and BASDAI.

Results: When comparing AS patients and controls pair wise, we observed on average a $50 \%$ reduction of TFH (CD3+CD4+CXCR5+) cells among CD45+ lymphocytes in PBMCs from patients $(p=0,000008)$. Furthermore, a $20-30 \%$ reduction among memory/plasma cells (CD19+CD27+CD38+ and CD19+CD27+CD38-) among CD45+ lymphocytes in PBMCs from patients $(p=0,002$ and $p=0,007$ respectively). For female patients a correlation between TFH and ESR $\left(R_{s}=\right.$ $0,551 \mathrm{p}=0,022)$ was observed. Moreover, negative correlations between the two B cell subsets (CD19+CD27+CD38+ and CD19+CD27+CD38-) and ESR were observed for female patients $\left(R_{s}=-0,476 p=0,053\right.$ and $R_{s}=-0,522 p=0,032$ respectively).

Conclusion: TFH cells was reduced in AS patients and this reduction correlated with a reduction in differentiated (CD27+CD38+ and CD27+CD38-) B cells. In 
addition, the inflammation marker ESR was negatively correlated with TFH as well as with the differentiated $B$ cell subsets in female patients. Our observations indicates a role of the humoral immune response in AS.

Disclosure of Interests: None declared

DOI: 10.1136/annrheumdis-2021-eular.785

\section{OP0025 CD209+/CD14+ DENDRITIC CELLS ARE ENRICHED AND ACTIVATED AT THE SITE OF INFLAMMATION AND ARE MODULATED BY JAK/STAT SIGNALLING}

V. Marzaioli ${ }^{1,2}$, A. Floudas ${ }^{1,2}$, M. Canavan ${ }^{1,2}$, S. Wade ${ }^{1,2}$, K. Murray ${ }^{2}$, R. Mullan ${ }^{3}$, D. Veale ${ }^{1,2}$, U. Fearon ${ }^{1,2}$. Trinity Biomedical Sciences Institute, 1 Molecular Rheumatology, Dublin, Ireland; ${ }^{2}$ Centre for Arthritis and Rheumatic Diseases, SVUH, EULAR Centre of Excellence for Rheumatology, Dublin, Ireland;

${ }^{3}$ Adelaide and Meath Hospital, Department of Rheumatology, Dublin, Ireland

Background: Dendritic cells (DCs) are a heterogeneous population of professional antigen-presenting cells which are at the interface between innate and adaptive immunity. A specific subset of DCs is known to derive from monocyte and has a key role in inflammation and infection.

Objectives: This study aimed to characterize the phenotype and function of a distinct $\mathrm{CD} 209^{+} / \mathrm{CD} 14^{+} \mathrm{DC}$ subset in the periphery and at the site of inflammation in patients with rheumatoid (RA) and psoriatic arthritic (PsA), in addition to examining the effect Tofacitinib and TNF inhibitor on their development.

Methods: Peripheral blood and synovial fluid mononuclear cells (PBMC and SFMC) were isolated by Ficoll density gradient from healthy subject $(\mathrm{HC})$, and patients with RA and PsA. Single cell synovial tissue suspension (ST) from RA and PsA patients were also established using enzymatic/mechanical digestion. PBMC, SFMC and ST were analysed by flow cytometry to identify the CD $209^{+} /$ CD14 ${ }^{+}$DC subset, its frequency and the cell surface expression of chemokines receptors (CCR6, CCR7, CXCR3, CXCR4 and CXCR5) and activation markers (CD40 and CD80). In addition, PBMC were stimulated with different TLR (LPS, CPG, R848, Poly I:C) and intracellular staining for IL 12, TNF $\alpha$, IL $1 \beta$ and IL6 was performed by flow cytometry. Lineage negative cells (CD3/CD19/CD56") were stimulate with GMCSF/IL4 in the presence or absence of the JAK/STAT inhibitor Tofacitinib or the TNF inhibitor Humira, and the CD209+/CD14+ DC development was evaluated by flow cytometry.

Results: We identified, for the first time, a distinct CD209+/CD14+ DC population in PBMC of patients with RA and PsA, with similar frequency across the groups. However, when PBMC were stimulated with TLRs, an increase of IL12 and TNF $\alpha$ was observed in RA and PSA PBMC when compared to HC. Interestingly, this distinct DC population was significantly enriched at the site of inflammation, in both SFMC and ST, displaying a more mature phenotype, evident by the observed significant increase in CD40 and CD80 expression. SPICE analysis further identified differential expression and co-expression of chemokine receptors at the periphery of RA and PsA patients, when compared to the HC. Furthermore synovial tissue single cell analysis from RA/PsA demonstrated a unique chemokines receptors profile demonstrating increased single expression and co-expression of CXCR3 and CXCR5 compared to periphery. Finally, we have previously observed that JAK/STAT is involved in monocyte-derived dendritic cells population development $(1,2)$, therefore we performed CD3, CD19 and CD56 depletion of RA/PsA PBMC followed by stimulation with GMCSF/IL4, to spike the Mo-DC population, in the presence of Tofacitinib or Humira. Interestingly, we observed that JAK/STAT inhibition, but not TNF inhibitor, reduced the generation and development of CD209+/ CD14+ DC.

Conclusion: We identify for the first time a distinct monocyte-derived DC population characterized as $\mathrm{CD} 209^{+} / \mathrm{CD} 14^{+}$in the periphery of RA and PsA patients. This population was enriched at the site of inflammation and displayed a unique chemokine receptor profile and activation markers, suggesting that these cells are already activated in the periphery of IA patients, and are recruited and further activated in the inflamed joint. In addition, we showed that the CD209 ${ }^{+} / C D 14^{+}$DC development is regulated by JAK/STAT signalling, but not TNF inhibition.

REFERENCES:

[1] Marzaioli V, Canavan M, Floudas A, et al. Monocyte-Derived Dendritic Cell Differentiation in Inflammatory Arthritis Is Regulated by the JAK/STAT Axis via NADPH Oxidase Regulation. Front. Immunol. 2020;11:1406.

[2] Marzaioli V, Hurtado-Nedelec M, Pintard C, et al. NOX5 and p22phox are 2 novel regulators of human monocytic differentiation into dendritic cells. Blood. 2017;130(15):1734-1745.

Acknowledgements: The authors also wish to thank all the patients who volunteered to participate into this study and the funding

Disclosure of Interests: Viviana Marzaioli: None declared, Achilleas Floudas: None declared, Mary Canavan: None declared, Siobhan Wade: None declared, Kieran Murray: None declared, Ronan Mullan: None declared, Douglas Veale Speakers bureau: Abbvie, Janssen, Novartis, MSD, Pfizer, UCB, Consultant of: Abbvie, Janssen, Novartis, MSD, Pfizer, UCB, Grant/research support from:
Janssen, Abbvie, Pfizer, UCB, Ursula Fearon Speakers bureau: Abbvie, Grant/ research support from: Janssen, Abbvie, Pfizer, UCB

DOI: 10.1136/annrheumdis-2021-eular.2122

\section{\begin{tabular}{l|l} 
OP0026 & IGF1R DEPENDENT CELL INTERACTION AND
\end{tabular} REGULATION OF AUTOANTIBODY PRODUCTION IN RHEUMATOID ARTHRITIS}

S. Erdogan ${ }^{1}$, M. Erlandsson ${ }^{1,2}$, N. Oparina ${ }^{1}$, C. Lundquist ${ }^{1}$, C. Wasen ${ }^{1}$, M. Svensson ${ }^{1}$, M. Bemark ${ }^{3}$, K. M. Andersson ${ }^{1}$, M. I. Bokarewa ${ }^{1,2}$. 'Gothenburg University, Rheumatology and Inflammation Research, Göteborg, Sweden; ${ }^{2}$ Sahlgrenska University Hospital, Rheumatology Clinic, Göteborg, Sweden; ${ }^{3}$ Göteborg University, Microbiology and Immunology, Göteborg, Sweden

Background: The insulin-like growth factor 1 receptor (IGF1R) signalling mediates numerous developmental processes acting through downstream adaptor molecules IRS $1 / 2$, which activate Akt and inhibit the family of forkhead box class O (FoxO). Inhibition of IGF1R signalling alleviates rheumatoid arthritis (RA) (Erlandsson et al., 2017), however, the role of IGF1R signalling in the regulation of immune function is poorly understood.

Objectives: To investigate the link between IGF1R signalling and antigen presentation in experimental arthritis.

Methods: Arthritis was induced by immunising Balb/c mice with methylated bovine serum albumin ( $\mathrm{mBSA}, \mathrm{n}=18$ ) and $\mathrm{DBA} / 1$ mice with type II collagen (CII, $\mathrm{n}=18$ ). The mice were treated with a synthetic IGF1R inhibitor NT157 or with short hairpin RNA targeting IGF1R (shIGF1R) from the day prior to immunisation. Controls were treated with cyclodextrine vehicle/ non-targeting (nt)RNA, respectively. Flow cytometry was used for spleen cell phenotype. Antibody levels were measured by ELISA. Immunohistochemistry (IHC) of spleen was performed fo assessment of marginal zone (MZ) and location of $\mathrm{pS}^{612} \mathrm{RS} 1+$ and $\mathrm{pS}^{256} \mathrm{FoxO} 1+$ cells. IHC images were acquired by fluorescent confocal microscopy, and analysed using ZEN2009 and Cell Profiler soft ware.

Results: The inhibition of IGF1R resulted in an $80 \%$ increase in MZ area in NT157 treated mice compared to controls $(p=0.0001)$. This was supported by a significant increase of CD21+ $(p=0.034)$ and CD23+ cell populations $(p=0.00059)$, both among the CD19+ B cells and antigen-presenting MHCII+CD19-cells, implying that IGF1R expression regulates the populations of $\mathrm{MZ}$ and follicular cells. Additionally, there was a strong positive correlation between the decrease of IGF1R+ and ICOSL+ population on CD21+ cells $(r=0.70, p=0.0071)$, which retained them in the $\mathrm{MZ}$ and prolonged communication with macrophages. Insufficient feedback from ICOSL- B cells limited expression of CXCR5 on CD4 cells. The IHC analysis displayed that, IGF1R inhibition led to abundance of inactivate $\mathrm{pS}^{612} \mathrm{IRS} 1+$ and $\mathrm{pS}^{256} \mathrm{FoxO} 1+$ cells within the $\mathrm{MZ}$, compared with controls $(\mathrm{p}=0.0002)$. Alongside the increase of IgM+B cell population $(p=0.0022)$, we observed significant increase in number of antigen-presenting $F 4 / 80+$ cells $(p=0.043)$ and MARCO expression $(p=0.043)$ after IGF1R intervention. Finally, the NT157- treated mice displayed a significant pleiotropic increase in IgM autoantibody production, with anti-CCP IgM ( $p=0.027), R F-\operatorname{lgM}(p=0.0085)$, anti-DNA IgM $(p=0.066)$ and in total $\operatorname{IgM}(\mathrm{p}=0.027)$ levels, which correlated positively with $\mathrm{pS}^{256} \mathrm{FoxO} 1^{+}$cells $(r=0.51$ $\mathrm{p}=0.03)$. Levels of IgG were not changed.

Conclusion: We show that IGF1R signalling is important for immune cell communication after antigen challenge. IGF1R controls ICOSL dependent trafficking of $B$ cells through the $M Z$ and facilitates interaction with $T$ cells. Retention of $B$ cells in the MZ tips the balance from $T$ cell to macrophage-dependent processes, which permits the formation of autoantibody producing B cells.

\section{REFERENCES:}

[1] Erlandsson, M., et.al., 2017. IGF-1R signalling contributes to IL-6 production and $\mathrm{T}$ cell dependent inflammation in rheumatoid arthritis. Biochimica et Biophysica Acta (BBA) - Molecular Basis of Disease, 1863(9), pp.2158-2170. Disclosure of Interests: None declared DOI: 10.1136/annrheumdis-2021-eular.2440

\section{OP0027 \\ AS-RELATED TCR BETA CLONOTYPES ARE PRESENT IN DIFFERENT INFLAMED TISSUES OF PATIENTS WITH SPONDYLOARTHROPATHIES}

E. Komech ${ }^{1}$, A. Barinova ${ }^{1}$, E. Shmidt ${ }^{2}$, T. Korotaeva ${ }^{3}$, A. Koltakova ${ }^{3}$, N. Nikitina ${ }^{4}$, E. Belousova ${ }^{4}$, M. Shapina ${ }^{5}$, S. Rodionovskaya ${ }^{6}$, I. Nikishina ${ }^{7}$, S. Vahlyarskaya ${ }^{8}$, D. Atarshchikov ${ }^{9}$, E. Klescheva ${ }^{10}$, D. Chudakov ${ }^{1,11}$, S. Lukyanov ${ }^{12}$, I. Zvyagin ${ }^{1,11}$. ${ }^{1}$ Pirogov Russian National Research Medical University, Institute of Translational Medicine, Center for Precision Genome Editing and Genetic Technologies for Biomedicine, Moscow, Russian Federation; ${ }^{2}$ Pirogov Russian National Research Medical University, City Clinical Hospital \#1, Moscow, Russian Federation; ${ }^{3}$ Nasonova Research Institute of Rheumatology, Lab of spondyloarthritis and psoriatic arthritis, Moscow, Russian Federation; ${ }^{4}$ Moscow Regional Research Clinical Institute, Gastroenterology Department, Moscow, Russian Federation; ${ }^{5}$ State Scientific Center of Coloproctology, Gastroenterology Department, Moscow, Russian Federation; ${ }^{6}$ Children's Clinical Hospital of 